\title{
ESTUDOS DE CASO COMO OPÇÃO DE PESQUISA EMPÍRICA EM OPERAÇÕES
}

João Mário Csillag joao.mario.csillag@fgv.br

FGV-EAESP

Ricardo Martins martins@cepead.face.ufmg.br UFMG-CEPEAD

Marcos André Mendes Primo marcos.primo@ufpe.br UFPE-PROPAD

\section{INTRODUÇÃO}

A área de Operações e Gestão da Cadeia de Suprimentos (O\&SCM) tem sido fortemente influenciada pela pesquisa quantitativa, especialmente por modelos de pesquisa operacional e modelos estatísticos oriundos da aplicação de surveys. A nossa área difere de outras áreas de pesquisa pelo fato de incluir tanto elementos físicos quanto humanos (DREJER, BLACKMON, VOSS, 1998). Nesse contexto, a pesquisa quantitativa não consegue captar todas as variáveis que envolvem o atual ambiente de produção, marcado pelas condicionantes externas, flexibilidade na produção, relações interorganizacionais, alta competitividade e aspectos humanos. Focalizando as publicações de três dos maiores jornais da área no período 19802006, Pilkington e Meredith (2009) afirmam que tem havido uma mudança no foco de pesquisa de tópicos específicos e táticos para tópicos mais gerais e estratégicos, passando pela inclusão de novos métodos e técnicas de pesquisa.

De acordo com Rocha (2005), a aplicação mais óbvia da pesquisa qualitativa seria no estudo de processos os quais se caracterizariam pela "existência de grande número de fatores intervenientes, em que as relações entre os fatores são complexas e desconhecidas", o que é típico da área de O\&SCM. Apesar do potencial de aplicação da pesquisa qualitativa, existem problemas para a sua difusão na área de O\&SCM. Além da pouca familiaridade dos pesquisadores da área com os procedimentos metodológicos da pesquisa qualitativa, existem a desconfiança quanto à cientificidade dessa pesquisa, especialmente pelo domínio do paradigma positivista dos pesquisadores na área, e o receio das possibilidades diminutas de publicação internacional utilizando esse tipo de pesquisa.
A fim de discutir esses pontos, a Divisão de Gestão de Operações e Logística (GOL) da Associação Nacional dos Cursos de Pós-Graduação (ANPAD) decidiu convidar o Prof. Tom Choi, editor do renomado Journal of Operations $\mathrm{Ma}$ nagemennt (JOM), para dar uma palestra sobre o tema no último encontro do EnANPAD (realizada no Rio de Janeiro em 5 de setembro de 2011). De acordo com o palestrante, o enfoque nos estudos de caso é um método qualitativo promissor na área de O\&SCM e que tem dado significativa contribuição para a elaboração de teorias no campo, em especial nas áreas de manufatura estratégica (BARRATT, CHOI, LI, 2011). Trabalhos no Brasil apontam o estudo de caso como a abordagem qualitativa mais adotada pelos pesquisadores no campo, talvez por ser considerado mais confortável para estudos em organizações e nos estudos em administração da produção e ope- 
rações (MARCHI, DELLAGNELO, ERDMANN, 2011).

O estudo de caso é uma abordagem de pesquisa que procura atender as questões do tipo "como" e "por que" tal fenômeno ocorre em determinado contexto (YIN, 1994).

Um estudo de caso é uma estória de um passado ou fenômeno corrente, desenhado a partir de múltiplas fontes de evidência. Pode incluir dados de observação direta e entrevista sistemática como também arquivos públicos e particulares. Em realidade, todo fato relevante à cadeia de eventos descrevendo o fenômeno é um dado potencial num estudo de caso, desde que o contexto seja importante (LEONARD-BARTON, 1990)
ESTUDOS DE CASOS EM OPERAÇÕES E GESTÃO DA CADEIA DE SUPRIMENTOS

Durante a palestra no EnANPAD, o Prof. Tom Choi afirmou que tem havido um aumento de publicações usando estudos de caso qualitativos nos principais jornais internacionais da área de O\&SCM (ver Gráfico 1). Esse aumento tem sido relevante nos periódicos da área focados em pesquisa empírica, especialmente nos dois periódicos com maior prestígio internacional nessa área, a saber: o JOM (do qual o palestrante é editor) e o International Journal of Operations and Production Management (IJOPM - ver Gráfico 2). A princípio, estudos de caso podem ser utilizados para diferentes tipos de finalidade de pesquisa: exploração, construção de teorias, teste de teorias e extensão/refinamento de teorias (VOSS, TSIKRIKTSIS, FRHLICH, 2002). A discussão conduzida pelo Prof. Choi no encontro realizado em 2011 destacou o foco da aplicação dessa abordagem nos estudos de caso indutivos (CHOI e HONG, 2002; WU e CHOI, 2005) e nos estudos de caso dedutivos (PINFIELD, 1986), com o objetivo, respectivamente, de construção de teoria ou de testar uma teoria existente.

De acordo com o Prof. Choi, a opção pelo uso dos estudos indutivos tem contribuído mais para a evolução dos estudos de casos na nossa área (vide Gráfico 3). Entretanto, como editor do JOM, o Prof. Choi nota que há, ainda, pouco rigor na aplicação do método de estudos de casos, principalmente

\section{Gráfico 1 - Tendência de publicação de estudos de caso}

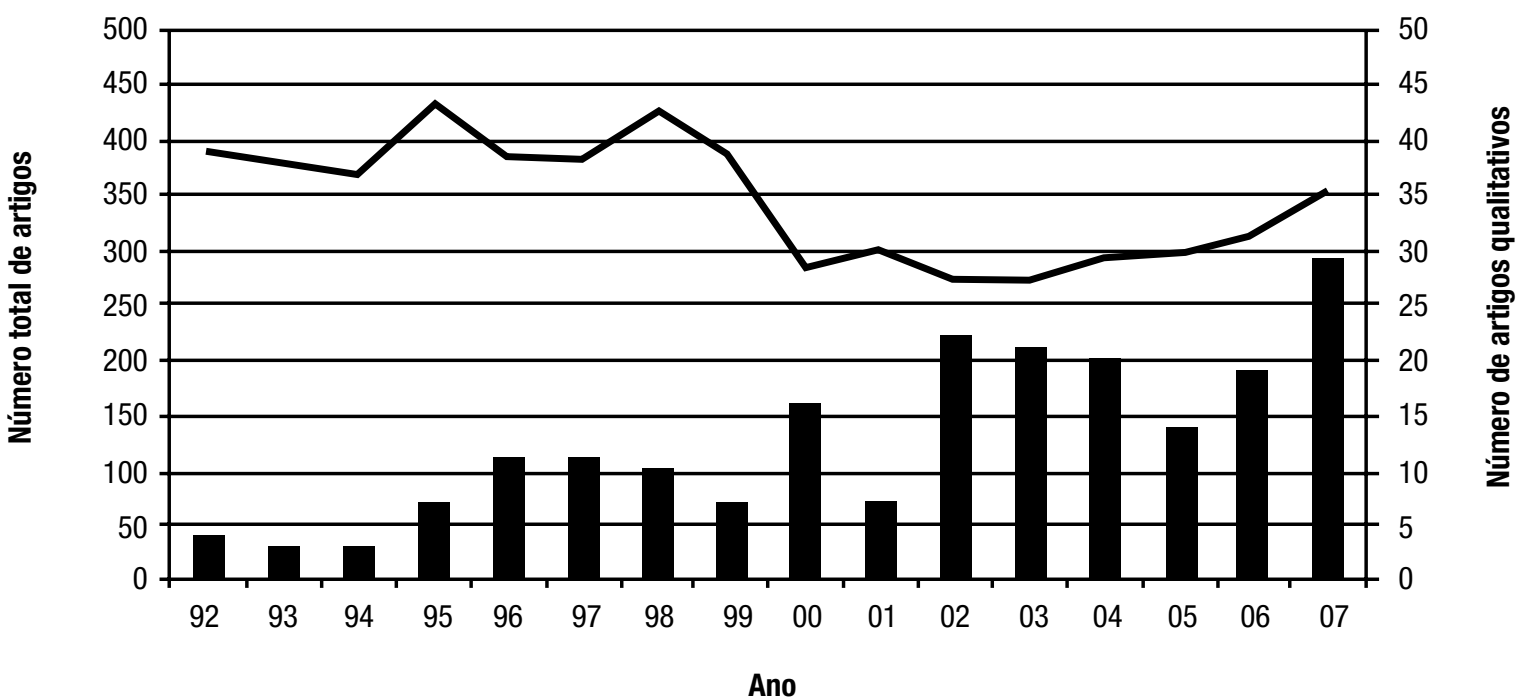

Estudo de casos qualitativos Total de artigos

Fonte: Material extraído da palestra de Tom Choi, no EnANPAD (realizada no Rio de Janeiro em 5 de Setembro de 2011) 
nos estudos de caso dedutivos. Ainda segundo o professor e editor, as maiores críticas quanto à utilização de estudos de caso em O\&SCM seriam a ausência de justificativa adequada para a utilização desse método como alternativa a outras opções metodológicas e o pouco rigor na utilização dessa abordagem de pesquisa. Quanto ao rigor dos procedimentos metodológicos, o uso de triangulação na composição dos dados raramente vai além da comparação dos dados de informantes com os de outras fontes, tais como documentos. A triangulação iterativa expandiria a noção tradicional de triangulação utilizando estudos de caso existentes para possibilitar diversidade representativa. Isso poderia ser alcançado pelo emprego de numerosas técnicas indutivas, comparando conjecturas para rever a literatura e conduzindo experimentos mentais, examinando diversos sites de pesquisa, metodologias e perspectivas teóricas por meio de estudos de casos (LEWIS, 1998).

Especificamente em relação aos estudos de caso indutivos, outras deficiências comuns encontradas nos trabalhos que adotam essa abordagem na área de O\&SCM são:

- A ausência de construção de teoria em contraposição à mera descrição de fenômenos;

- Viés do pesquisador quando entra no campo com grande interesse, mas propenso a interpretar os fatos como um advogado, e não como observador neutro (VOSS, TSIKRIKTSIS, FRHLICH, 2002);

- Má definição das questões de pesquisa e unidades de análise;

- Pouca consideração do papel das teorias existentes em explicar o fenômeno estudado;

- Problemas relacionados com a amostragem, seleção dos casos e número de casos utilizados;

- Problemas na organização e análise dos dados;

- Problemas na apresentação dos resultados da pesquisa.

Em relação à preocupação dos pesquisadores nacionais quanto às possibilidades de publicação internacional de trabalhos utilizando os estudos de caso, o Prof. Choi estimula a submissão de artigos empíricos ao JOM guiados por teorias em O\&SCM. Há interesse específico em estudos que avancem o conhecimento sistemático de "como" e "por que" alguns fenômenos ocorrem e/ou afetam o desempenho em operações ou "como" e "por que" há uma rela-

\section{Gráfico 2 - Publicação de estudos de caso nos principais jornais de O\&SCM}

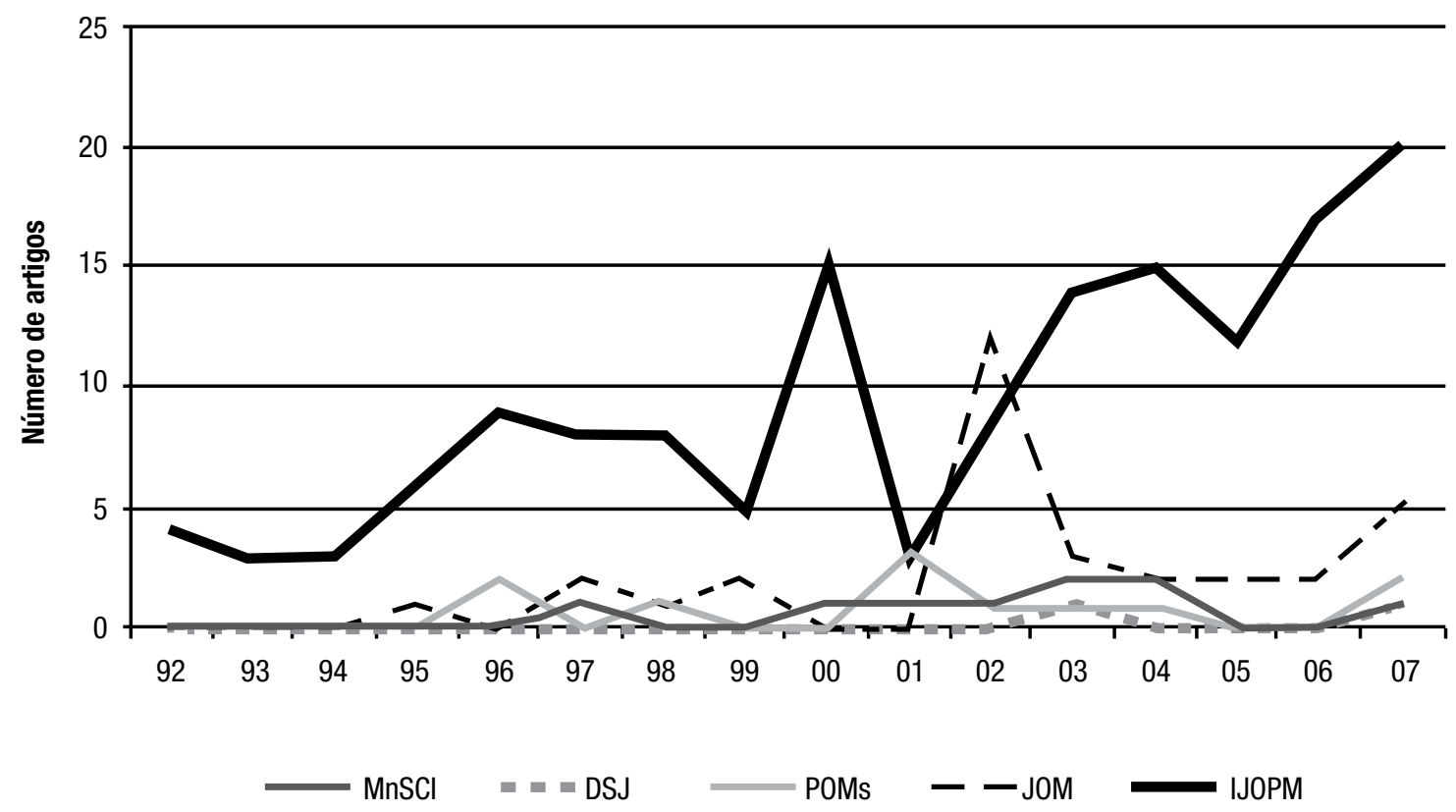

Fonte: Material extraído da palestra de Tom Choi, no EnANPAD (realizada no Rio de Janeiro em 5 de Setembro de 2011) 
ção entre dois ou mais fenômenos nessa área. Tendo em vista esse posicionamento do editor de um jornal de prestígio em O\&SCM (atualmente, o fator de impacto do JOM é 4,382), isso é muito estimulante para quem faz pesquisa qualitativa na área.

\section{CONCLUSÕES}

Analisando as questões abordadas na apresentação do Prof. Choi, podemos ressaltar os seguintes pontos relacionados à pesquisa qualitativa em O\&SCM:

- Pesquisa exploratória é usualmente qualitativa e, em alguns casos, pesquisa qualitativa em si pode ser um novo desenvol- vimento teórico (SINGHAL e SINGHAL, 2012). Essas pesquisas exploratórias qualitativas antecedem pesquisas quantitativas explanatórias;

- Estudos de caso não devem ser vistos apenas como uma metodologia apropriada para compreensão e para estágios preliminares de desenvolvimento de teoria. Devido a sua riqueza observacional, também permitem refutações e extensões de conceitos existentes (STUART e outros, 2002);

- Para a fase de análise, Eisenhardt (1989) propõe duas etapas: análise dos dados do caso e pesquisa de padrões entre casos. Entretanto, essa última fase nem sempre é devidamente utilizada com uma boa análise cruzada dos casos;
- Apesar da boa vontade do editor do JOM, trabalhos qualitativos originais que tenham produzido impacto não foram publicados em nenhum periódico líder na área de O\&SCM nas últimas cinco décadas, presumivelmente porque esses periódicos não acolhem bem artigos que não incluam intensas análises matemáticas e estatísticas (SINGHAL e SINGHAL, 2012).

- O rigor em pesquisa é obtido com a validade interna e externa. A validade interna depende da situação específica do contexto considerado. Cabe ao pesquisador decifrar a cadeia de causas para a situação específica. A validade externa depende do contexto, de empresa a empresa, de momen-

\section{Gráfico 3 - Publicação de estudos de caso indutivos versus dedutivos}

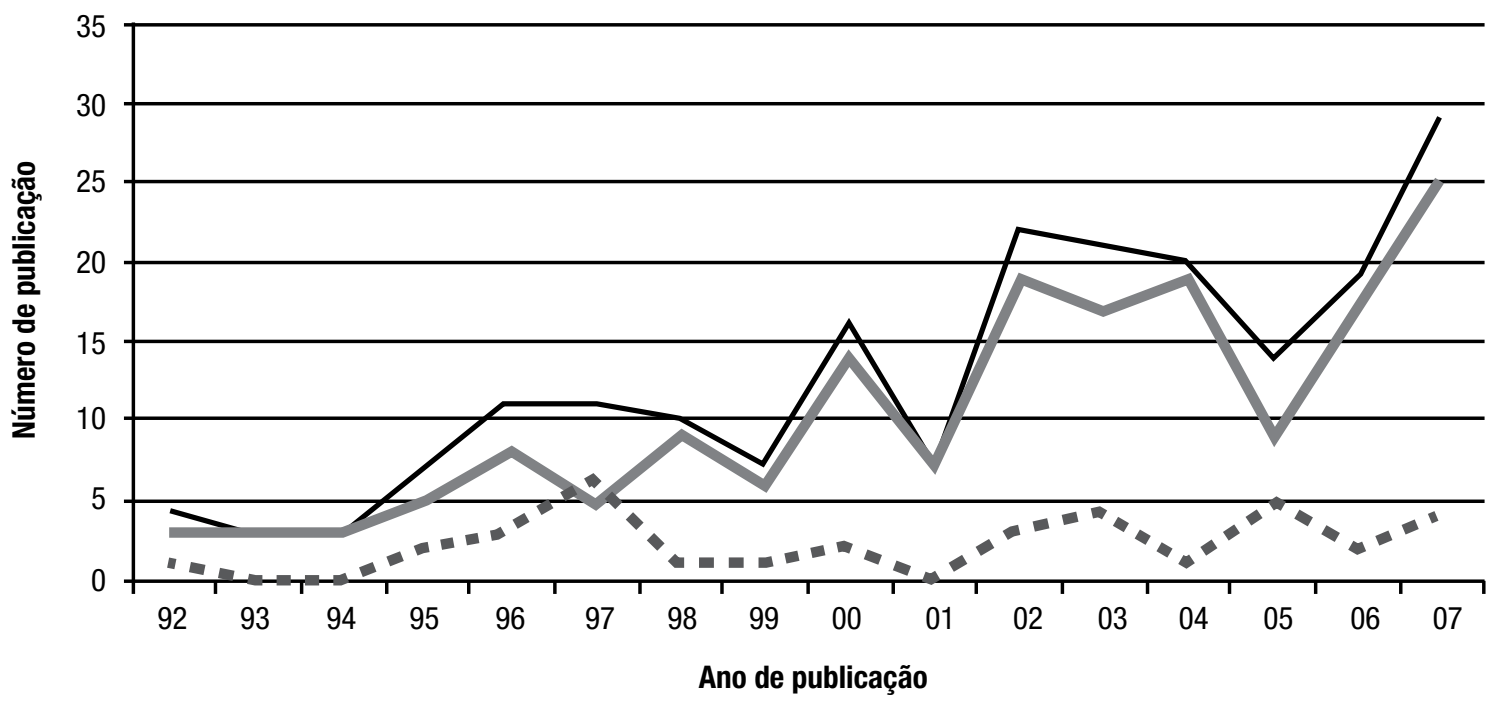

— Total de artigos qualitativos $\quad$ Indutivo = = Dedutivo 
to a momento para a mesma empresa, de um executivo a outro. Logo, deve-se procurar perspectivas múltiplas, cada uma baseada em verificação empírica (SINGHAL e SINGHAL, 2012);

- Quanto aos problemas de análise e redução de dados, já existem procedimentos metodológicos para auxiliar a pesquisa qualitativa. Huberman e Miles (1983) apresentaram um trabalho interessante para redução de dados em pesquisa qualitativa. Os dados compostos de muitas palavras podem ser reduzidos, seja por meio de codificação ou graficamente;

- A utilização de softwares de análise de texto (exs.: Atlas TI, Nudist), apesar de reduzir a complexidade do trato das informações, pressupõe diretrizes do pesquisador quanto à análise, redução e codificação dos dados. Ou seja, diferentemente das análises estatísticas convencionais, em que procedimentos para essas etapas estão difundidos e assimilados, os pesquisadores da área necessitam de um conhecimento profundo sobre procedimentos metodológicos de pesquisa qualitativa para se beneficiar dessas ferramentas.

A importância da pesquisa qualitativa para o progresso da área de O\&SCM, em especial os estudos de caso, é inegável. Entretanto, para vencer as resistências dos bons periódicos internacionais quanto a esse tipo de abordagem qualitativa, é necessário investir no rigor dos procedimentos metodológicos, assim como ajustar o foco da área, ainda muito concentrado na solução de problemas específicos para o desenvolvimento de teoria, que possa ajudar a explicar fenômenos que ocorrem nos diversos processos relativos a operações e cadeia de suprimentos.

\section{FÓRUM GESTÃO DE OPERAÇÕES}

Com base em uma análise de todos os artigos apresentados no XIV Simpósio de Administração da Produção, Logística e Operações Internacionais, promovido pelo Departamento de Administração da Produção e de Operações da Escola de Administração de Empresas de São Paulo, Fundação Getulio Vargas, em 2011, foram pré-selecionados oito artigos com potencial para publicação na $R A E$. Essa pré-seleção resultou na submissão de versões aperfeiçoadas de seis artigos para avaliação pelo fórum. Para que a avaliação fosse a mais imparcial possível, solicitamos que cada artigo fosse avaliado por dois pesquisadores ad hoc experientes e renomados de instituições de fora do(s) estado(s) de origem do(s) autor(es) do artigo. Após cuidadosa avaliação dos pareceres dos avaliadores e atendimento das suas sugestões pelos autores, foram selecionados três artigos para compor essa edição do Fórum SIMPOI/RAE. Entre os seis artigos selecionados para avaliação, apenas um deles utilizou o método quantitativo, e os demais foram qualitativos, sendo que quatro deles apresentaram estudos de casos. De certa forma, a discussão apresentada aqui sobre uso de estudos de casos em O\&SCM ilustra a prática de pesquisa dos autores que submeteram seus artigos ao Fórum SIMPOI/RAE.
O artigo "Redes de empresas e seus efeitos sobre o turismo", dos autores Angela Cabral Flecha, Alcides Volpato Castro e Silva, José Paulo Alves Fusco e Américo Tristão Bernardes, apresenta uma interessante proposta de utilização da análise de redes sociais (ARS) no estudo do turismo. Na área de operações, predomina a análise da governança nas redes interorganizacionais, e a abordagem da ARS ainda é pouco explorada na nossa área. Com base no mapeamento da rede formada pelos atores individuais que operacionalizam os serviços de comercialização e desenvolvimento de negócios, foi possível esboçar os desenhos das redes e avaliar a densidade dos elos entre fornecedores e clientes, assim como sua importância relativa no contexto do turismo. Como resultado, um conjunto de indicadores foi apresentado para avaliar o alinhamento competitivo dos componentes da rede de atores de turismo.

No artigo "Recursos, controle e autonomia na gestão internacional de serviços de uma empresa de TI e subsidiárias", Daniel Scumparim e Mário Sacomano Neto analisaram as características da Gestão de Serviços Globalmente Integrada (GSGI) de uma multinacional de tecnologia de informação e subsidiárias, explorando questões paradoxais do controle central e da autonomia local. Esse estudo abre perspectivas amplas de desenvolvimento no campo da internacionalização de empresas com foco nas operações de serviços, um segmento em franca expansão nacional e mundial.

Finalmente, o artigo "Competitividade das nações: análise da métrica utilizada pelo World Economic Forum", escrito por Luciano Castro 
de Carvalho, Luiz Carlos Di Serio e Marcos Augusto de Vasconcellos, busca analisar a métrica de competitividade dos países utilizando bases de dados de 2010 publicadas pelo World Economic Forum (WEF), que utiliza 12 pilares para estabelecer a condição competitiva de 133 países. A pesquisa conclui que a métrica utilizada pelo WEF é redundante, pois utiliza pilares que representam o mesmo constructo. Acrescenta que a prontidão tecnológica explica a competitividade do país em $86,5 \%$ e que a combinação entre estabilidade macroeconômica, qualidade do ensino superior e sofisticação dos negócios eleva esse percentual para $97,5 \%$. Outra contribuição do trabalho mostra duas métricas adicionais, a do Institute for Management Development (IMD) e a da United Nations Industrial Development Organizations (Unido), que nem sempre chegam a resultados similares quando utilizam as mesmas amostras.

Esperamos que os leitores da $R A E$ apreciem este Fórum sobre a Gestão de Operações e que ele amplie o esforço de melhoria contínua da qualidade da pesquisa em nossa área.

\section{REFERÊNCIAS}

BARRATT, M; CHOI, T. Y; LI, M. Qualitative case studies in operations management: trends, research outcomes, and future research implications. Journal of Operations Management, v. 29, n.4, p. 329-342, 2011.

CHOI, T; HONG, Y. Unveiling the structure of supply networks: case studies in Honda, Acura, and DaimlerChrysler. Journal of Operations Management, v. 20, n. 5, p. 469-493, 2002.
DREJER, A; BLACKMON, K; VOSS, C. Worlds apart? - a look at the operations management area in the US, UK and Scandinavia. Scandinavian Journal of Management, v. 16 n.1, p. 45-66, 2000.

EISENHARDT, K. M. Building theories from case study research. Academy of Management Review, v. 14, n. 4, p. 532-550, 1989.

HUBERMAN, A. M; MILES, M. Drawing valid meaning from qualitative data: some techniques of data reduction and display. Quality and Quantity, v. 17, n. 4, p. 281-339, 1983.

LEONARD-BARTON, D. A dual methodology for case studies: synergistic use of a longitudinal single site with replicated multiple sites. Organization Science, v. 1, n. 3, p. 248-266, 1990.

LEWIS, M. W. Iterative triangulation: a theory development process using existing case studies. Journal of Operations Management, v. 16, n. 4, p. 455-469, 1998.

MARCHI, J. J; DELlaGNELO, E. H. L; ERDMANN, R. H. Pesquisa qualitativa no campo da administração da produção e operações: enlaces e oportunidades de aplicação. II Encontro de Ensino e Pesquisa em Administração e Contabilidade, João Pessoa, 2011.

PILKINGTON, A; MEREDITH, J. The evolution of the intellectual structure of operation management - 19802006: a citation/co-citation analysis. Journal of Operations Management, v. 27, n.3, p. 185-202, 2009.

PINFIELD, L. A field evaluation of perspectives on organizational decision making. Administrative Science Quarterly, v. 31, n. 3, p. 365-388, 1986.
ROCHA, A. Métodos qualitativos em administração: usos e abusos. Informativo ANPAD, editorial, n. 6, 2005.

SINGHAL, K; SINGHAL, J. Opportunities for developing the science of operations and supply-chain management. Journal of Operations Management, v. 30, n. 3, p. 245-252, 2012.

STUART, I. e outros. Effective case research in operations management: a process perspective. Journal of Operations Management, v. 20, n.5, p. 419-433, 2002.

VOSS, D; TSIKRIKTSIS, N; FRHLICH, $M$. Case research in operations management. International Journal of Operations \& Production Management, v. 22, n. 2, p. 195-219, 2002.

WU, Z; CHOI, T. Supplier-supplier relationships in the buyer-supplier triad: building theories from eight case studies. Journal of Operations Management, v. 24, n. 1, p. 27-52, 2005.

YIN, R. K. Case study research: design and methods. 2nd ed. Newbury Park: Sage, 1994. 